ORIGINAL PAPER

\title{
A Rare CD 5-Positive SUbGroup of Diffuse large B-CELL LYMPHOMA - CLINICAL, MORPHOLOGICAL AND immunophenotypic features in Polish patients
}

\author{
Nina Woźnialis ${ }^{1}$, Beata Gierej ${ }^{1,2}$, Lidia Popeawska ${ }^{3}$, Mateusz Ziarkiewicz ${ }^{4}$, \\ Elżbieta Kulczycka ${ }^{1}$, Bogna Ziarkiewicz-Wróblewska ${ }^{1,2}$
}

\begin{abstract}
${ }^{1}$ Department of Pathology and Laboratory Diagnostics, Maria Skłodowska-Curie Institute and Oncology Centre, Warsaw, Poland

${ }^{2}$ Department of Pathology, Center for Biostructure Research, Medical University of Warsaw, Warsaw, Poland ${ }^{3}$ Department of Lymphoid Malignancies, Maria Skłodowska-Curie Institute and Oncology Centre, Warsaw, Poland

${ }^{4}$ Department of Hematology, Oncology and Internal Medicine, Medical University of Warsaw, Warsaw, Poland
\end{abstract}

\begin{abstract}
The aim of the study was to assess the incidence of $\mathrm{CD} 5$-positive diffuse large B-cell lymphoma (DLBCL) in the Polish population and to describe its morphologic and clinical characteristics. The study included 36 patients with CD5-positive DLBCL, diagnosed and treated in the Maria Skłodowska-Curie Institute and Oncology Centre, Warsaw, Poland and the Medical University of Warsaw, Poland in the years 2002-2013. The control group consisted of 28 patients with CD5-negative DLBCL. CD5-positive DLBCL accounted for 6.26\% of all DLBCL cases diagnosed in the Maria Skłodowska-Curie Institute and Oncology Centre in the years 2008-2012. The incidence is comparable to other European countries, lower than noted in Japan and higher than in the US. Patients with CD5-positive DLBCL, in comparison to the $\mathrm{CD} 5$-negative group, were characterized by: (1) older age ( $\geq 60$ vs. younger) and worse general status (ECOG $\geq 2$ vs. $<2$ ), (2) lower frequency of complete remission (CR), (3) higher expression of unfavorable prognostic factors (BCL2, FOXP1, CD44) and MMP-9, and (4) lower expression of CD 30, cyclin D1, cyclin D3 and TIMP-2.
\end{abstract}

Key words: NHL, CD5-positive DLBCL, prognostic factors.

\section{Introduction}

Diffuse large B-cell lymphoma (DLBCL) is the most frequent aggressive non-Hodgkin's lymphoma (NHL). It occurs predominantly in the elderly, typically follows an aggressive clinical course and requires prompt medical treatment [1].

CD5-positive DLBCL remains an insufficiently studied, relatively new entity, which accounts for $5-10 \%$ of the DLBCL population [2]. The mean age of patients with CD5-positive DLBCL is 70 years, with a moderate female predominance $(1: 1.2)$, in contrast to CD5-negative DLBCL, which prevails in males $(1.4: 1)$. The majority of patients with CD5-positive DLBCL have a high International Prognostic Index (IPI) score [3, 4, 5].

Relatively few literature reports suggest worse clinical outcomes in patients with CD5-positive DLBCL, resulting from resistance to chemotherapy. It remains debatable whether CD5-positive DLBCL constitutes a separate clinical entity or merely an immunophenotypic variant of DLBCL with adverse prognostic features. The limited knowledge in this field stimulates further research on this lymphoma [6]. 
Immunohistochemistry (IHC) plays a crucial role in the diagnosis and subclassification of DLBCL. There are multiple prognostic factors described in DLBCL, which not only facilitate the diagnosis, but also support proper treatment decisions in specific patients. Favorable prognostic factors include, among others, the expression of CD30 [7], BCL6 [8] and a low proliferative index [9]. Adverse prognostic factors comprise the expression of cyclin D2 [10], CD138 [8], CD44 [11], p53 [12], BCL2 [13, $14,15]$, and more importantly, the co-expression of BCL2 and CD10 [16]. The role of cyclin D3 [17, 18], FOXP1 $[13,19]$ and CD10 as prognostic factors in patients with DLBCL is not fully elucidated on the basis of the available literature $[20,21,22]$.

C-MYC protein overexpression is a new adverse prognostic factor in DLBCL. Identification of this antigen helps determine DLBCL patients with poor prognosis [23]. Other recently described antigens associated with unfavorable clinical outcomes and shorter overall survival of DLBCL patients are MAGE-A3 [24], CD43 [25] and PD-L1 [26].

Extracellular matrix metalloproteinases (MMPs) belong to the family of zinc endopeptidases. Their expression and function is detected in the majority of human cells [27]. The main role of MMPs is the degradation of extracellular matrix (ECM), which promotes cell migration and stimulates release of growth factors, proapoptotic factors, chemokines and cytokines [28]. In physiologic conditions, MMPs take part in multiple important processes such as angiogenesis, apoptosis, embryogenesis, tissue remodeling, healing of injuries or scar formation. The excessive activity of MMPs is observed in various diseases, including malignancies, Alzheimer disease, cardiovascular disorders, autoimmune diseases, osteoarthritis, psoriasis and liver cirrhosis [29]. MMP activity is regulated at various stages of their biosynthesis. Moreover, tumor infiltrating inflammatory cells, stromal cells and malignant cells secrete growth factors and cytokines which modulate the activity of MMPs. MMP expression is also tightly controlled by $\alpha-2$-macroglobulin, a non-specific metalloproteinase inhibitor, and by endogenous tissue inhibitors of metalloproteinases (TIMPs), which include TIMP-1, TIMP-2, TIMP-3 and TIMP-4 [30].

MMP-2 and MMP-9 play an important role in the development of DLBCL and are associated with progression of the disease. MMP-2 and MMP-9 degrade type IV collagen, which is a part of basement membranes. They are involved in lymphoma processes through pro-inflammatory cytokines, chemokines and other proteins which are released during degradation of the ECM, e.g. osteopontin and interleukin-6 [31]. MMP-2, MMP-9 and TIMP-2 together comprise a crucial proteolytic axis which regulates numerous aspects of neoplasia in DLBCL. TIMP-2 creates complexes with MMP-2. Lymphoma patients with the highest levels of the aforementioned complexes have a greater risk of relapse [32].

The aim of this study was to compare the clinical characteristics of patients with $\mathrm{CD} 5$-positive DLBCL with the CD5-negative DLBCL subgroup and to precisely assess the detailed immunophenotype of CD5-positive DLBCL with the special consideration of prognostic factors and extracellular matrix metalloproteinases, as well as proteins connected with the enzymatic function.

\section{Material and methods}

Eight hundred and forty-six cases of diffuse large B-cell lymphoma (DLBCL) were diagnosed in the Maria Skłodowska-Curie Institute and Oncology Center in the years 2008-2012. In 53 cases CD5 expression was detected. Paraffin-embedded tissue blocks were available for further investigation only in 15 cases out of this group, because material sent for consultation was returned to primary sites, and some diagnoses were made on the basis of flow cytometry alone. The remaining cases were treated and diagnosed in the Medical University of Warsaw in the years 20022013. Finally, the study material included 36 specimens of CD5-positive DLBCL (27 lymph nodes, 5 stomachs, 2 brains, 1 intestine, 1 testis) obtained from 19 females (52.8\%) and 17 males (47.2\%) aged between 29 and 87 years (mean age 69) treated and diagnosed in the Maria Skłodowska-Curie Institute and Oncology Centre and the Medical University of Warsaw in the years 2002-2013. The control group comprised 28 specimens of CD5-negative DLBCL (23 lymph nodes, 3 stomachs, 1 intestine, 1 liver) obtained from 14 women $(50 \%)$ and 14 men $(50 \%)$ aged between 24 and 82 years (mean age 58.5).

\section{Histological procedures}

Tissue samples for histological analysis were fixed in $4 \%$ formalin buffer, embedded in paraffin blocks according to standard procedure and then cut into slices 3-4 $\mu \mathrm{m}$ thick. Slices were subsequently stained with hematoxylin and eosin (HE).

\section{Immunohistochemical procedures}

Immunohistochemical staining (IHC) was performed in the Autostainer Link 48 automatic staining system manufactured by Dako or manually at room temperature $\left(20-25^{\circ} \mathrm{C}\right)$. Staining protocols were based on standard methodology and manufacturers' recommendations in the case of the following antibodies: CD20, CD3, CD5, CD10, Ki67, MUM1, BCL2, BCL6, cyclin D1, CD138 and CD30. In the case of several antibodies (VEGF, CD44, HIF1 $\alpha$, MMP-2, TIMP-2, MMP-9, cyclin D3, FOXP1 and SOX11) an 
Table I. Individual IHC staining protocols developed for studied antigens

\begin{tabular}{ccccc}
\hline Antibody & Chosen dilution & Antigen Retrieval & $\begin{array}{c}\text { Linker } \\
(15 \mathrm{~min})\end{array}$ & Primary antibody incubation time \\
\hline anti-VEGF & $1 / 25$ & $\mathrm{pH} 9.0$ & - & $20 \mathrm{~min}$ \\
\hline anti-CD44 & $1 / 50$ & $\mathrm{pH} 6.0$ & - & $20 \mathrm{~min}$ \\
\hline anti-MMP-2 & $1 / 30$ & $\mathrm{pH} 9.0$ & yes & $30 \mathrm{~min}$ \\
\hline anti-TIMP-2 & $1 / 50$ & $\mathrm{pH} 9.0$ & - & $20 \mathrm{~min}$ \\
\hline anti-MMP-9 & $1 / 100$ & $\mathrm{pH} 9.0$ & - & $30 \mathrm{~min}$ \\
\hline anti-cyclin D3 & $1 / 50$ & $\mathrm{pH} 9.0$ & - & $20 \mathrm{~min}$ \\
\hline anti-FOXP1 & $1 / 300$ & $\mathrm{pH} 9.0$ & - & $20 \mathrm{~min}$ \\
\hline anti-SOX11 & $1 / 100$ & $\mathrm{pH} 9.0$ & - & \\
\hline
\end{tabular}

individual staining protocol was developed, including selection of buffer for antigen retrieval, concentration and duration of incubation (Table I). The staining protocol with MMP-2 additionally included the linker reagent to enhance the IHC reaction.

Detection of the primary antibodies bound with the antigens listed above was obtained using secondary antibodies conjugated with horseradish peroxidase (HRP). Visualization of this reaction was acquired using 3,3'-diaminobenzidine (DAB). The last step was the counterstaining with hematoxylin to visualize the nuclei.

Basically, the assessment of staining was based on estimation of both the amount of cells with a positive reaction (0-100\%) and the staining intensity $(0$, $+,++,+++)$. The reactions with VEGF, MMP-2, MMP-9 and TIMP-2 were evaluated according to the Remmele score, whose final result is the product of the proportion score assigned for the amount of positive cells ( $0-4$ points) and the reaction intensity score (0-3 points), known as the Immunoreactive Score (IRS), ranging from 0 to 12 points [33].

\section{Statistical analysis}

Statistical analysis was performed using the IBM SPSS Statistics 19 software. The Fisher exact test with $2 \times 2$ contingency tables was used to assess the significance of the differences in distribution of sex, elevated LDH level, general symptoms (A/B), IPI risk groups, response to treatment, ECOG performance status $\geq 2$, clinical stage III-IV, age over 60 years and number of extranodal sites involved between CD5-positive and CD5-negative DLBCL. The significance of the differences between IHC staining scores in patients with CD5-positive and CD5-negative DLBCL was assessed using the Mann-Whitney test. The results were regarded as statistically significant if the $\mathrm{P}$ value was below 0.05 for each of the aforementioned tests.

\section{Results}

CD5-positive DLBCL accounted for $6.26 \%$ of all DLBCL cases diagnosed in the Maria Skłodowska-Curie Institute and Oncology Center in the years 2008-2012. Full clinical data were collected for 58 patients with the exception of six cases of CD5-positive DLBCL, which were treated in various oncologic centers in Poland and the tissue material was sent for consultation only.

Among the analyzed clinical data, only three parameters differed significantly between study groups (Table II). Age $>60$ years and ECOG performance status $\geq 2$ were more frequently observed in CD5-positive DLBCL in comparison to patients with CD5-negative DLBCL (Fisher exact test $\mathrm{p}=$ 0.006 and $p=0.034$, respectively). As a result, patients with CD5-positive DLBCL were more frequently classified in the high-risk group according to the International Prognostic Index (IPI). Moreover, response to treatment defined as complete remission (CR) was significantly better in CD5-negative DLBCL than in CD5-positive DLBCL (Fisher exact test $\mathrm{p}=$ 0.03). The remaining parameters did not differ significantly between the study groups.

Significant differences between DLBCL patients grouped according to $\mathrm{CD} 5$ expression were found only in the case of selected IHC stains (Tables III, IV). The Mann-Whitney test showed higher expression of BCL2 ( $p=0.000)$, FOXP1 $(p=0.000)$, CD44 ( $\mathrm{p}=0.003)$, and MMP-9 $(\mathrm{p}=0.045)$ in patients with $\mathrm{CD} 5$-positive DLBCL in comparison to CD5-negative cases (Figs. 1-3). On the other hand, the expression of CD30 ( $\mathrm{p}=0.000)$, cyclin D1 
Table II. Clinical parameters in patients with CD5-positive DLBCL and CD5-negative DLBCL

\begin{tabular}{|c|c|c|c|c|}
\hline Parameter & & $\begin{array}{c}\operatorname{DLBCL} \mathrm{CD} 5(+) \\
(\%)\end{array}$ & DLBCL CD5(-) (\%) & P value \\
\hline \multirow[t]{2}{*}{ Sex } & $\mathrm{F}$ & 52.80 & 50 & \multirow[t]{2}{*}{0.512} \\
\hline & M & 47.20 & 50 & \\
\hline Age $>60$ & & 86.7 & 53.6 & 0.006 \\
\hline $\mathrm{LDH}>\mathrm{ULN}$ & & 56.7 & 71.40 & 0.185 \\
\hline Stage III-IV & & 46.7 & 71.4 & 0.49 \\
\hline \multirow[t]{2}{*}{ General symptoms } & A & 50.0 & 32.1 & \multirow[t]{2}{*}{0.133} \\
\hline & $\mathrm{B}$ & 50.0 & 67.9 & \\
\hline ECOG PS $\geq 2$ & & 43.3 & 17.9 & 0.034 \\
\hline \multirow[t]{3}{*}{ IPI } & low & 16.7 & 25.0 & \multirow[t]{3}{*}{0.573} \\
\hline & intermediate & 60.0 & 60.7 & \\
\hline & high & 23.3 & 14.3 & \\
\hline Extranodal involvement $\geq 2$ sites & & 26.7 & 25.0 & 0.562 \\
\hline \multirow[t]{4}{*}{ Response to treatment } & $\mathrm{CR}$ & 63.3 & 78.6 & 0.03 \\
\hline & $\mathrm{PR}$ & 6.7 & 7.1 & 0.593 \\
\hline & SD & 6.7 & 10.7 & 0.38 \\
\hline & PD or death & 23.3 & 3.6 & 0.06 \\
\hline
\end{tabular}

$C R$ - complete remission; $P R$ - partial remission; $S D$ - stable disease; $P D$ - progressive disease; $L D H$ - lactate dehydrogenase; ULN - upper limit of normal; ECOG

PS - Eastern Cooperative Oncology Group Performance Status scale; IPI - International Prognostic Index

Table III. Mean percentage of positive IHC staining cells in CD5-positive DLBCL and CD5-negative DLBCL with statistical assessment

\begin{tabular}{cccc}
\hline ANTIGEN & DLBCL CD $5(+)$, MEAN & DLBCL CD $5(-)$, MEAN & P VALUE \\
\hline CD20 & 0.96 & 0.94 & 0.095 \\
\hline CD3 30.16 & 0.16 & 0.418 \\
\hline CD5 & 0.16 & 0.00 & 0.000 \\
\hline CD10 & 0.96 & 0.22 & 0.259 \\
\hline Ki67 & 0.21 & 0.79 & 0.070 \\
\hline MUM1 & 0.85 & 0.54 & 0.362 \\
\hline BCL2 & 0.66 & 0.51 & 0.000 \\
\hline BCL6 & 0.84 & 0.49 & 0.057 \\
\hline Cyclin D1 & 0.58 & 0.08 & 0.000 \\
\hline Cyclin D3 & 0.00 & 0.36 & 0.037 \\
\hline CD138 & 0.24 & 0.00 & 1.000 \\
\hline FOXP1 & 0.00 & 0.47 & 0.000 \\
\hline CD44 & 0.83 & 0.75 & 0.003 \\
\hline SOX11 & 0.89 & 0.00 & 1.000 \\
\hline CD30 & 0.00 & 0.16 & 0.000 \\
\hline
\end{tabular}

$(\mathrm{p}=0.000)$, cyclin D3 $(\mathrm{p}=0.037)$, and TIMP-2 $(\mathrm{p}=0.000)$ was higher in the CD5-negative DLBCL group. The results of the remaining stains were comparable between the study groups.

\section{Discussion}

The $4^{\text {th }}$ edition of the WHO classification (2008) recognizes 3 immunohistochemical subgroups of DLBCL, not otherwise specified (NOS): germinal cen- 
Table IV. Mean results of IHC staining according to the Remmele score in CD5-positive DLBCL and CD5-negative DLBCL with statistical assessment

\begin{tabular}{cccc}
\hline ANTIGEN & DLBCL CD5(+), MEAN & DLBCL CD5(-), MEAN & P VALUE \\
\hline MMP-2 & 0.58 & 0.93 & 0.667 \\
\hline MMP-9 & 2.06 & 1.30 & 0.045 \\
\hline TIMP-2 & 0.72 & 7.07 & 0.000 \\
\hline VEGF & 1.08 & 0.96 & 0.188 \\
\hline
\end{tabular}

ter B-cell (GCB), non-GCB and, the least frequent, CD5-positive DLBCL, which accounts for 5-10\% of DLBCL, NOS. In Texas, USA, the frequency of this lymphoma has been estimated to be 5\% (13/260) of the DLBCL population [34], in a Scandinavian study it was $7 \%(9 / 125)$ [35], whereas in Japan it was as high as $10 \%(24 / 240)$ [36]. In the Polish population analyzed in the present study, CD5-positive DLBCL accounted for $6.26 \%$ (53/846) of all DLBCL cases.

CD5-positive DLBCL is considered as a subtype of DLBCL with aggressive clinical characteristics. It occurs in the elderly with female predominance and more frequent extralymphatic localization. In comparison to the CD5-negative DLBCL population, patients with CD5-positive DLBCL are characterized by a more advanced clinical stage at diagnosis, worse general condition, higher LDH level and more frequent central nervous system involvement, as well as a worse response to chemotherapy [37].

The results of the present study confirm higher incidence of CD5-positive DLBCL in the elderly ( $>60$ years) and worse general condition of these patients (ECOG performance status $\geq 2$ ) in comparison to the CD5-negative DLBCL population. These are two of five prognostic factors used to assess the risk group according to the International Prognostic Index (IPI). The remaining parameters used to assess IPI (clinical stage according to Ann Arbor classifica-

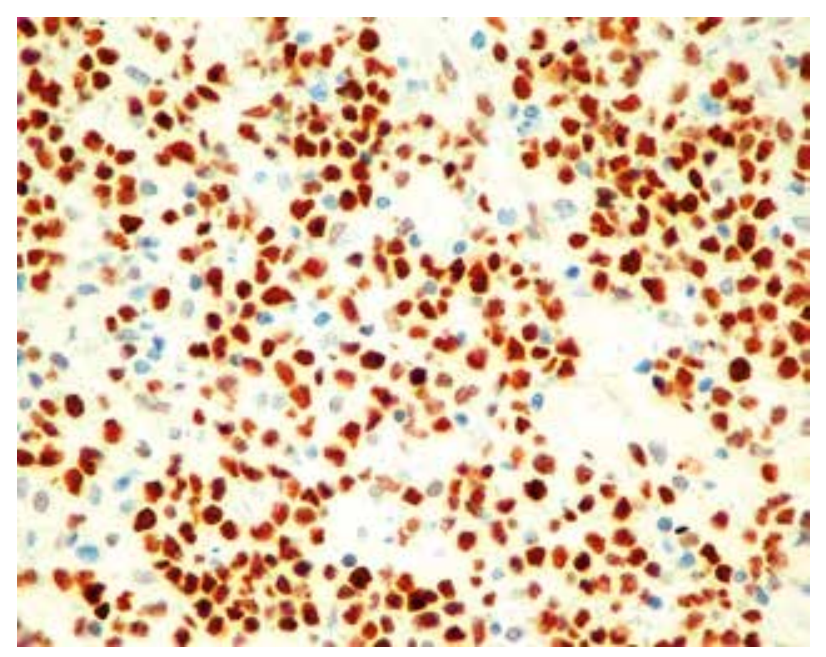

Fig. 2. Immunohistochemical staining with anti-FOXP1 in a case of CD5-positive DLBCL (magnification $400 \times$ ) tion, number of extranodal sites involved and serum LDH activity) did not differ significantly between the analyzed groups.

Patients with CD5-positive DLBCL were characterized by a significantly lower CR rate in comparison to the group with CD5-negative DLBCL, which indicates its aggressive course and unfavorable prognosis.

The results obtained by Ennishi et al. confirm the poor clinical outcomes in CD5-positive DLBCL. The

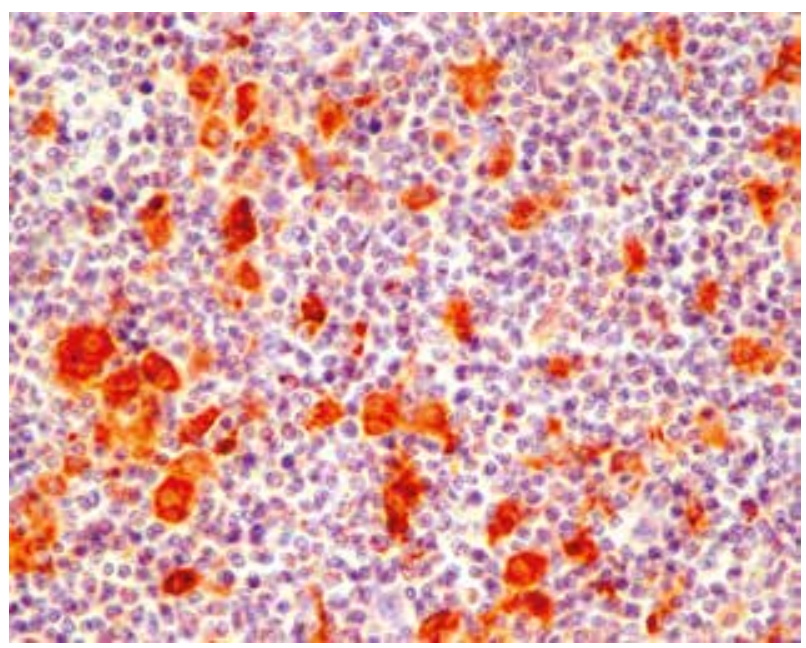

Fig. 1. Immunohistochemical staining with anti-MMP-9 in a case of CD5-positive DLBCL (magnification $400 \times$ )

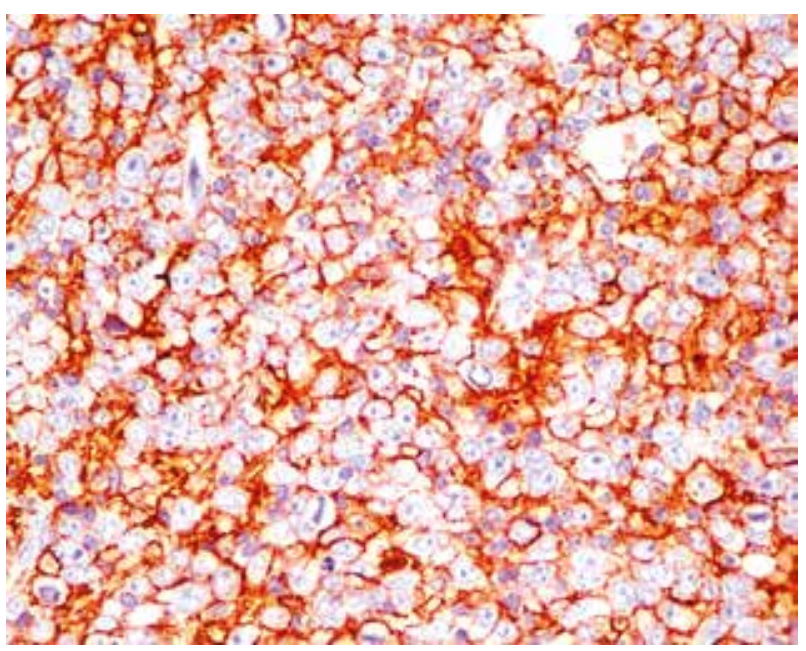

Fig. 3. Immunohistochemical staining with anti-CD44 in a case of CD5-positive DLBCL (magnification $400 \times$ ) 
overall survival (OS) and event-free survival (EFS) reported by these authors were $91 \%$ and $73 \%$, respectively, in the CD5-negative group, and $45 \%$ $(\mathrm{p}=0.001)$ and $18 \%(\mathrm{p}<0.001)$, respectively, in the CD5-positive group [5]. The studies by Chan et al. [3] and Zheng et al. [4] also confirm this observation.

The results of immunohistochemical analyses performed by the authors of the present study also indicate the aggressive behavior of the disease. It is characterized by higher expression of unfavorable prognostic factors and lower expression of favorable prognostic factors. Włodarska et al. documented intensive FOXP1 expression in DLBCL [38]. Moreover, it is more often observed in the non-GCB subtype. Some authors reported that the expression of FOXP1 on lymphoid cells was associated with worse clinical outcomes [19]. In the present study, the expression of FOXP1 was noted both in CD5-positive DLBCL and CD5-negative DLBCL. However, the intensity of staining was different, being significantly higher in CD5-positive DLBCL (Mann-Whitney test, $\mathrm{p}=0.000$ ). This observation is a notion towards the association between FOXP1 expression and clinical course of the lymphoma. However, Nyman et al. suggest that the prognostic significance of FOXP1 is doubtful, especially in contrast to BCL2 [13], whose unfavorable impact in DLBCL remains firmly documented $\{13,14,15]$, and may be further enhanced in cases with co-expression of CD10 [16]. High BCL2 expression, found in $40-60 \%$ of the DLBCL population, leads to uncontrolled proliferation of lymphoid cells [14]. In line with these results, the staining performed in the present study showed significantly higher BCL2 expression in patients with CD5-positive DLBCL in comparison to the CD5-negative group (Mann-Whitney test, $\mathrm{p}=0.000$ ).

The neoplastic cells in DLBCL frequently show positive immunostaining for CD44, an adhesion factor facilitating the dissemination of malignant cells in the body and thus the progression of the disease. According to the reported data, its expression is higher in non-GCB in comparison to the GCB subtype, which may partially explain the unsatisfactory treatment outcomes in the former type of DLBCL [11]. The CD44 protein plays a major role in promoting angiogenesis and disease progression. It simulates proliferation and motility of neoplastic cells, enables metalloproteinase activity, and enhances the development of tumor vasculature [39]. Inagaki et al. described the association between high CD44 expression and shorter OS in patients with DLBCL [40]. In the present study, high expression of CD44 was documented in the CD5-positive DLBCL population. The majority of cases showed CD44 expression in $100 \%$ of malignant cells. In contrast, CD 5 -negative
DLBCL cases showed significantly weaker CD44 expression (Mann-Whitney test, $\mathrm{p}=0.003$ ).

The presence of $\mathrm{CD} 30$ on the surface of neoplastic cells was found in $32 \%$ of cases in CD5-negative DLBCL (9/28), whereas it was completely absent in CD5-positive DLBCL (0/36) (Mann-Whitney test, $\mathrm{p}=0.000)$. Hu et al. [7] concluded that CD30 expression is a favorable prognostic factor in DLBCL patients. The results of immunostaining with anti-CD30 antibody performed in the present study further confirm the worse prognosis in patients with CD5-positive DLBCL.

Angiogenesis is a complex process of new blood vessel formation on the basis of the existing vasculature. The first step is the proteolytic degradation of the extracellular matrix by metalloproteinases. Subsequently, migration and proliferation of the endothelial cells ensues, and finally, formation and maturation of blood vessels occur. The first scientist to indicate the potential role of angiogenesis in cancer development was Judah Folkman in 1971 [41]. There are multiple factors with pro- and antiangiogenic properties. Depending on the domination of one or the other group, induction or inhibition of angiogenesis ensues. Formation of new vessels is stimulated by various cytokines, including vascular endothelial growth factor (VEGF), which inhibits apoptosis and stimulates proliferation of endothelial cells, as well as increasing permeability of vascular walls [42].

There are conflicting literature reports concerning VEGF expression and its clinical impact in DLBCL. Zhu et al. [43] observed that VEGF expression is associated with worse response to chemotherapy and unfavorable prognosis in patients with DLBCL. However, Gratzinger et al. [44] suggest that increased tumor vascularization is associated with shorter survival of patients with DLBCL. Nevertheless, the authors found that a high level of VEGF expression in malignant cells did not correlate with the degree of regional vascularization. Similarly, the results of immunohistochemical reactions with anti-VEGF performed in the present study did not confirm any association between the level of VEGF expression and the clinical behavior of DLBCL. Both study groups, CD5-positive and CD5-negative DLB$\mathrm{CL}$, were characterized by comparable expression of this protein (Mann-Whitney test, $\mathrm{p}=0.188$ ).

Extracellular matrix metalloproteinases play an important role in the process of angiogenesis. MMPs degrade the stroma, creating space for new malignant cells and budding blood vessels. MMPs also induce angiogenesis through release of multiple growth factors and proangiogenic factors from the surface of cells [45]. Pathologic processes in the body are usually accompanied by a decrease of TIMP activity. This in turn leads to increased activity and enhanced expression of MMPs in the malignant tissue. Excessive 
MMP synthesis with concomitant TIMP deficiency results in intensification of tissue degradation and, finally, in deterioration of the neoplastic disease. This process promotes migration of malignant cells and predisposes to the development of metastatic foci [46]. Increased TIMP expression is frequently found both in tumor stroma and in malignant cells. There are data to confirm the thesis that overexpression of TIMPs in malignant cells reduces their aggressiveness and ability to form metastases. Among others, Qi et al. documented that TIMP-3 inhibits tumor growth and angiogenesis intensity [47].

The present study showed that the level of MMP-2 expression was comparable in patients with $\mathrm{CD} 5$-positive and CD5-negative DLBCL (Mann-Whitney test, $\mathrm{p}=0.67)$. On the other hand, MMP-9 expression was higher in CD5-positive DLBCL (Mann-Whitney test, $\mathrm{p}=0.045)$. It may be associated with adverse clinical course of this lymphoma subtype, because MMP-9 releases multiple factors from the extracellular matrix, which promote growth and dissemination of the malignant disease. One of them is KIT ligand, which induces angiogenesis and thus facilitates dissemination of neoplastic cells [48]. Moreover, significantly higher TIMP-2 expression was noted on malignant cells in CD5-negative DLBCL, in comparison to CD5-positive DLBCL (Mann-Whitney test, $\mathrm{p}=0.000)$. Unarguably, it is associated with lower ability to metastasize in this subtype of lymphoma, as TIMPs inhibit metalloproteinase activity.

The majority of authors have reported similar observations to those made in the present study, namely that the expression of MMPs in malignant cells, including MMP-9, is associated with unfavorable prognosis in patients with aggressive NHL, such as DLBCL [49, 50, 51]. Feng et al. reported that the expression of MMP-9 was higher in DLBCL in clinical stage III and IV in comparison to clinical stage I and II [52]. Nevertheless, there are reports suggesting that the expression of MMP-2, MMP-9, TIMP-1 and TIMP-2 has no influence on survival in DLBCL patients [53].

The knowledge about the mechanism of action of MMPs and development of their inhibitors may significantly influence future treatment strategies, creating an alternative to conventional chemotherapy and radiotherapy. The aim of such therapy would be to inhibit their enzymatic activity in target tissues. Synthetic MMP inhibitors could potentially restrain tumor growth, as well as its ability to develop metastases [46]. MMP inhibitor are currently being assessed in clinical trials [54]. Preclinical studies on tumor models gave promising results. Unfortunately, the results of clinical studies in cancer patients are still unsatisfactory [27]. Studying the mechanism of action of metalloproteinases and their inhibitors may be necessary in future to create novel therapeutic modalities in DLBCL.

\section{Conclusions}

1. The expression of $\mathrm{CD} 5$ on DLBCL cells was associated with poor prognosis factors (BCL2, FOXP1, CD44) and MMP-9.

2. The expression of CD5 on DLBCL was noted in the minority of these patients $(6.26 \%)$ and characterized a group with different clinical features, course and prognosis.

3. Patients with CD5-positive DLBCL in comparison to patients with CD5-negative DLBCL were more frequently classified in the high-risk group according to the International Prognostic Index (IPI), were older ( $>60$ years) and were in worse clinical condition (ECOG performance status $\geq 2$ ).

Further studies on metalloproteinases and their inhibitors in the future may provide important prognostic information and create a basis for targeted therapy in a selected group of patients with lymphomas.

\section{The authors declare no conflict of interest.}

\section{References}

1. Friedberg JW. Diffuse Large B-Cell Lymphoma. Hematol Oncol Clin North Am 2008; 22: 941-949.

2. Swerdlow SH, Campo E, Harris NL, et al. WHO Classification of Tumours of Haematopoietic and Lymphoid Tissues. $4^{\text {th }} \mathrm{ed}$. IARC Press, Lyon 2008; 9-15.

3. Chan AC, Chan JK. Diffuse Large B-cell Lymphoma. In: Hematopathology. Jaffe ES, Harris NL, Vardiman JV, et al. (eds.). Saunders Elsevier, Philadelphia 2011; 349-381.

4. Zheng Y, Ma XB, Jiang J, et al. CD5 expression is an adverse prognostic factor in diffuse large B-cell lymphoma. Zhonghua Binglixue Zazhi 2012; 41: 156-160.

5. Ennishi D, Takeuchi K, Yokoyama M, et al. CD5 expression is potentially predictive of poor outcome among biomarkers in patients with diffuse large B-cell lymphoma receiving rituximab plus CHOP therapy. Ann Oncol 2008; 19: 1921-1926.

6. Niitsu N, Okamoto M, Tamaru J, et al. Clinicopathologic characteristics and treatment outcome of the addition of rituximab to chemotherapy for CD5-positive in comparison with CD5-negative diffuse large B-cell lymphoma. Ann Oncol 2010; 21: 2069-2074.

7. Hu S, Xu-Monette ZY, Balasubramanyam A, et al. CD30 expression defines a novel subgroup of diffuse large B-cell lymphoma with favorable prognosis and distinct gene expression signature: a report from the International DLBCL Rituximab-CHOP Consortium Program Study. Blood 2013; 121: 2715-2724

8. Bodoor K, Matalka I, Hayajneh R, et al. Evaluation of BCL-6, CD10, CD138 and MUM-1 expression in diffuse large B-cell lymphoma patients: CD138 is a marker of poor prognosis. Asian Pac J Cancer Prev 2012; 13: 3037-3046.

9. Yoon DH, Choi DR, Ahn HJ, et al. Ki-67 expression as a prognostic factor in diffuse large B-cell lymphoma patients treated with rituximab plus CHOP. Eur J Haematol 2010; 85: 149157. 
10. Hans CP, Weisenburger DD, Greiner TC, et al. Expression of $\mathrm{PKC}$-beta or cyclin D2 predicts for inferior survival in diffuse large B-cell lymphoma. Mod Pathol 2005; 18: 1377-1384.

11. Tzankov A, Pehrs AC, Zimpfer A, et al. Prognostic significance of CD44 expression in diffuse large B cell lymphoma of activated and germinal centre $\mathrm{B}$ cell-like types: a tissue microarray analysis of 90 cases. J Clin Pathol 2003; 56: 747-752.

12. Leroy $\mathrm{K}$, Haioun $\mathrm{C}$, Lepage $\mathrm{E}$, et al. $\mathrm{p} 53$ gene mutations are associated with poor survival in low and low-intermediate risk diffuse large B-cell lymphomas. Ann Oncol 2002; 13: 11081115 .

13. Nyman H, Jerkeman M, Karjalainen-Lindsberg ML, et al. Bcl-2 but not FOXP1, is an adverse risk factor in immunochemotherapy-treated non-germinal center diffuse large B-cell lymphomas. Eur J Haematol 2009; 82: 364-372.

14. Schuetz JM, Johnson NA, Morin RD, et al. BCL2 mutations in diffuse large B-cell lymphoma. Leukemia 2012; 26: 1383 1390.

15. Song MK, Chung JS, Shin DH, et al. Prognostic significance of the $\mathrm{Bcl}-2$ negative germinal centre in patients with diffuse large B cell lymphoma treated with R-CHOP. Leuk Lymphoma 2009; 50: 54-61.

16. Xu Y, McKenna RW, Molberg KH, et al. Clinicopathologic analysis of CD10+ and CD10 - diffuse large B-cell lymphoma. Identification of a high-risk subset with coexpression of $\mathrm{CD} 10$ and bcl-2. Am J Clin Pathol 2001; 116: 183-190.

17. Shin HJ, Chung J, Park DY, et al. Prognostic significance of the cyclin D3 and the Ki-67 expression for predicting clinical outcome in diffuse large B-cell lymphoma. J Clin Oncol 2004; 22 (Suppl): 6714

18. Gumina MR, Xu C, Chiles TC. Cyclin D3 is dispensable for human diffuse large B-cell lymphoma survival and growth. Evidence for redundancy with cyclin E. Cell Cycle 2010; 9: 820-828.

19. Barrans SL, Fenton JA, Banham A, et al. Strong expression of FOXP1 identifies a distinct subset of diffuse large B-cell lymphoma (DLBCL) patients with poor outcome. Blood 2004; 104: 2933-2935.

20. Xu Y, McKenna RW, Molberg KH, et al. Clinical relevance of CD10 expression in de novo large B-cell lymphoma. Mod Pathol 2000; 13: 166

21. Fabiani B, Delmer A, Lepage E, et al. CD10 expression in diffuse large B-cell lymphomas does not influence survival. Virchows Archiv 2004; 445: 545-551.

22. Ohshima K, Kawasaki C, Muta H, et al. CD10 and Bcl10 expression in diffuse large B-cell lymphoma: CD10 is a marker of improved prognosis. Histopathology 2001; 39: 156-162.

23. Zhou K, Xu D, Cao Y, et al. C-MYC aberrations as prognostic factors in diffuse large B-cell lymphoma: A meta-analysis of epidemiological studies. PLoS One 2014; 9: 950-920.

24. Olarte I, Martinez A, Ramos-Penafiel C, et al. MAGE-A3 expression is an adverse prognostic factor in diffuse large B-cell lymphoma. Hematology 2011; 16: 368-372.

25. Ma XB, Zheng Y, Yuan HP, et al. CD43 expression in diffuse large B-cell lymphoma, not otherwise specified: CD43 is a marker of adverse prognosis. Hum Pathol 2015; 46: 593-599.

26. Boussiotis VA. Cell-specific PD-L1 expression in DLBCL. Blood 2015; 126: 2171-2172.

27. Visse R, Nagase H. Matrix metalloproteinases and tissue inhibitors of metalloproteinases. Structure, function, and biochemistry. Circ Res 2003; 92; 827-839.

28. Fink K, Boratyński J. The role of metalloproteinases in modification of extracellular matrix in invasive tumor growth, metastasis and angiogenesis. Postepy Hig Med Dosw (Online) 2012; 66; 609-628.

29. Gialeli C, Theocharis AD, Karamanos NK. Roles of matrix metalloproteinases in cancer progression and their pharmacological targeting. FEBS J 2011; 278: 16-27.
30. Löffek S, Schilling O, Franzke CW. Biological role of matrix metalloproteinases: a critical balance. Eur Respir J 2011; 38: 191-208.

31. Malaponte G, Hafsi S, Polesel J, et al. Tumor microenvironment in diffuse large B-cell lymphoma: Matrix metalloproteinases activation is mediated by osteopontin overexpression. Biochim Biophys Acta 2016; 1863: 483-489.

32. Pennanen H, Kuittinen O, Turpeenniemi-Hujanen T. Plasma MMP-2-TIMP-2 complex levels measured during follow-up predict a risk of relapse in patients with malignant lymphoma. Eur J Haematol 2008; 80: 46-54.

33. Remmele W, Stegner HE. Recommendation for uniform definition of an immunoreactive score (IRS) for immunohistochemical estrogen receptor detection (ER-ICA) in breast cancer tissue. Pathologe 1987; 8: 138-140.

34. Kroft SH, Howard MS, Picker LJ, et al. De novo CD5+ diffuse large B-cell lymphomas. A heterogeneous group containing an unusual form of splenic lymphoma. Hematopathology 2000; 114: 523-533.

35. Linderoth J, Jerkeman M, Cavallin-Ståhl E, et al. Immunohistochemical expression of $\mathrm{CD} 23$ and $\mathrm{CD} 40$ may identify prognostically favorable subgroups of diffuse large B-cell lymphoma. A Nordic Lymphoma Group Study. Clin Cancer Res 2003; 9: 722-728

36. Ogawa S, Yamaguchi M, Oka K, et al. CD21S antigen expression in tumour cells of diffuse large B-cell lymphomas is an independent prognostic factor indicating better overall survival. Br J Haematol 2004; 125: 180-186.

37. Jain P, Fayad LE, Rosenwald A. Recent advances in de novo CD5 + diffuse large B cell lymphoma. Am J Hematol 2013; 88: 798-802.

38. Wlodarska I, Veyt E, De Paepe P. FOXP1, a gene highly expressed in a subset of diffuse large B-cell lymphoma, is recurrently targeted by genomic aberrations. Leukemia 2005; 19 : 1299-1305

39. DeLisser HM. CD44: target for antiangiogenesis therapy. Blood 2009; 114: 5114-5115.

40. Inagaki $\mathrm{H}$, Banno S, Wakita A, et al. Prognostic significance of CD44v6 in diffuse large B-cell lymphoma. Mod Pathol 1999; $12 ; 546-552$

41. Cao Y, Langer R. A review of Judah Folkman's remarkable achievements in biomedicine. Proc Natl Acad Sci U S A 2008; 105: 13203-13205.

42. Ferrara N. Vascular endothelial growth factor: molecular and biological aspects. Curr Top Microbiol Immunol 1999; 237: $1-30$.

43. Zhu J, Diao Y, Jiang M. Significance of expression of VEGF in patients with diffused large B cell lymphoma. U.S. Chinese Journal of Lymphology and Oncology 2007; 6: 61-66.

44. Gratzinger D, Zhao S, Tibshirani RJ. Prognostic significance of VEGF, VEGF receptors, and microvessel density in diffuse large B cell lymphoma treated with anthracycline-based chemotherapy. Lab Invest 2008; 88: 38-47.

45. Curran S, Murray GI. Matrix metalloproteinases in tumour invasion and metastasis. J Pathol 1999; 189: 300-308.

46. Murphy G, Nagase H. Progress in matrix metalloproteinase research. Mol Aspects Med 2008; 29: 290-308.

47. Qi JH, Ebrahem Q, Moore N, et al. A novel function for tissue inhibitor of metalloproteinases-3 (TIMP-3): inhibition of angiogenesis by blockage of VEGF binding to VEGF receptor-2. Nat Med 2003; 9; 407-415.

48. Heissig B, Hattori K, Dias S, et al. Recruitment of stem and progenitor cells from the bone marrow niche requires MMP-9 mediated release of kit-ligand. Cell 2002; 109: 625-637.

49. Sakata KI, Satoh M, Someya M, et al. Expression of matrix metalloproteinase 9 is a prognostic factor in patients with non-Hodgkin lymphoma. Cancer 2004; 100: 356-365. 
50. Lenz G, Wright G, Dave SS, et al. Stromal gene signatures in large-B-cell lymphomas. N Engl J Med 2008; 359: 23132323.

51. Yoshida N, Oda M, Kuroda Y, et al. Clinical Significance of sIL-2R Levels in B-Cell Lymphomas. PLoS One 2013; 8: 1-10.

52. Feng YH, Wu LS, Su J, et al. Expression and significance of MMP-26, TIMP-4 and MMP-9 in diffuse large B-cell lymphoma cells. Zhongguo Shi Yan Xue Ye Xue Za Zhi 2013; 21: 1167-1172.

53. Kyllönen H, Pasanen AK, Kuittinen O, et al. Lack of prognostic value of MMP-9 expression and immunohistochemically defined germinal center phenotype in patients with diffuse large B-cell lymphoma treated with modern chemotherapy with or without CD20 antibody. Leuk Lymphoma 2009; 50: 1301-1307.

54. Fingleton B. MMP Inhibitor Clinical Trials - The Past, Present, and Future. In: The Cancer Degradome. Edwards D, et al (eds.). Springer Science + Business Media, LLC 2008; 759785 .

\section{Address for correspondence}

\section{Nina Woźnialis}

Department of Pathology and Laboratory Diagnostics

Maria Skłodowska-Curie Institute and Oncology Centre

Roentgena 5

02-781 Warsaw, Poland

e-mail: nina.woznialis@gmail.com 